Meta

Journal des traducteurs

Translators' Journal

\title{
Mini, micro, nano, pico... ou la petitesse en informatique
}

\section{Georges Lurquin}

Volume 26, numéro 4, décembre 1981

URI : https://id.erudit.org/iderudit/003525ar

DOI : https://doi.org/10.7202/003525ar

Aller au sommaire du numéro

Éditeur(s)

Les Presses de l'Université de Montréal

ISSN

0026-0452 (imprimé)

1492-1421 (numérique)

Découvrir la revue

Citer cet article

Lurquin, G. (1981). Mini, micro, nano, pico... ou la petitesse en informatique. Meta, 26(4), 381-384. https://doi.org/10.7202/003525ar d'utilisation que vous pouvez consulter en ligne.

https://apropos.erudit.org/fr/usagers/politique-dutilisation/ 
En informatique, la préhistoire, c'était, il y a quarante ans, les ordinateurs "complexes, servis avec difficulté», encombrants et fragiles, seulement accessibles en langage machine, puis en assembleur, et incompatibles. En quelques années, des progrès spectaculaires, notamment la découverte de nouveaux composants électroniques, ont amené des bouleversements qui constituent une 
véritable mutation, presque un changement de nature. «Aujourd'hui un microprocesseur de quelques millimètres de côté renferme la même puissance de traitement qu'un ordinateur qui représentait pourtant, il y a dix ou quinze ans, la quintessence technologique et qui aurait occupé une pièce entière. À puissance égale, un composant qui valait $350 \mathrm{~F}$ il y a dix ans vaut actuellement un centime. $\mathrm{Si}$ son prix avait connu une évolution comparable, la Rolls-Royce la plus luxueuse coûterait aujourd'hui un franc» (Nora-Minc).

Un mot caractérise l'évolution de l'informatique : miniaturisation; l'ordinateur devient le mini-ordinateur, l'informatique la mini-informatique, et la micro-informatique s'occupant de micro-ordinateurs constitue un immense domaine potentiel. On a miniaturisé les postes de radio par les transistors; on les a réduits à de plus petites dimensions sans que le fonctionnement soit modifié. On a microminiaturisé, réduit à l'extrême les dimensions et le poids des dispositifs électroniques. On subminiaturise lorsqu'on réalise des composants électroniques ou des circuits intégrés au moyen d'éléments extrêmement réduits de montages électroniques.

Pour exprimer la petitesse et ses degrés, la langue dispose d'opérateurs utilisés par les terminologies de nos diverses langues. Dérivant du latin et du grec, où ils signifient petit, très petit, extrêmement petit, ils se rencontrent de plus en plus dans les termes dont usent les informaticiens.

D'abord MINI. Emprunté au latin minus (= moindre, plus petit), mini- se rencontre dans de nombreux champs lexicaux, notamment dans ceux de la publicité, de la politique et des faits divers. "Il n'entre jamais dans un champ lexical marqué comme scientifique ${ }^{1}$. Il est devenu un élément préfixal de la langue commune (cf. minijupe, minibus). Les informaticiens ne l'ignorent pourtant pas et s'en servent pour désigner ce qui est plus petit que le normal.

Au début des années 60 sont nés les mini-ordinateurs. "Composés à l'origine d'une unité centrale miniaturisée et de structure relativement simple, ces matériels étaient dotés d'un logiciel sommaire et s'adressaient essentiellement aux domaines de l'instrumentation, du contrôle de processus, du calcul scientifique en bureau d'études, etc.» (Nora-Minc). Le traitement de l'information par les mini-ordinateurs est parfois désigné sous le nom de mini-informatique. Le mini-ordinateur est un compromis entre l'ordinateur central, noyau intégrateur d'un système distribué et le micro-ordinateur individuel. «Le mini-ordinateur est par vocation convivial, c'est-à-dire accessible à des unités locales (...) Le microréseau distribué qui en résulte réplique le macro-réseau des systèmes distribués ${ }^{2}$. En anglais : minicomputer (De Minicomputer, Minirechner), minicassette ( $E n$ mini-cassette, $D e$ Minicassette), minicontrôle, minidisque, minichaîne, etc.

Les sciences utilisent depuis toujours MICRO-, combiné avec un deuxième constituant grec: micrographie, microscope, microtome, microphone; de ce dernier, il devient bientôt l'abréviation. Les termes ainsi créés ne demandent pas à être traduits : $D e$ Mikrophon ou Mikrofon, $N l$ microfono, $I t, E l$ microfono, 1. Jean Peytard (1973): «De la diffusion d'un élément préfixal: mini-», Langue française, $\mathrm{n}^{\circ} 17$,
p. 18-30.

2. Bruno Lussato, le Dossier de la micro-informatique, p. 117. 
$R u$ mikrofon. Micro- se voit aussi associer un élément de la langue naturelle; ainsi, en métrologie, pour marquer la division d'une unité par un million, il se combine avec l'unité elle-même, sans trait d'union, lorsque le mot désignant l'unité commence par une consonne : micro-ampère ( = un millionième d'ampère, $\mu \mathrm{A}$ ), microseconde (un millionième de seconde, $\mu \mathrm{s}$ ), microfarad, microthermie, etc.; microhm (De Mikroohm).

Ce modèle de formation prédomine en informatique. Depuis quelques années sont apparus les microprocesseurs (En microprocessor, De Mikroprozessor). Constitués d'un circuit intégré, ils permettent de réaliser l'unité centrale d'un micro-ordinateur ( $E n$ microcomputer, De Mikrocomputer) et peuvent servir directement à cet usage ou être employés dans d'autres fonctions (machines à calculer de poche, unités de liaison de périphériques dans les grands ordinateurs, unités logiques et arithmétiques des terminaux intelligents, unités de commutation dans les réseaux). Les applications que peuvent avoir ces micro-ordinateurs ressortissent à la micro-informatique.

Un circuit de très petite dimension est un microcircuit (En microcircuit, $D e$ Mikroschaltung); une interruption brève de la transmission pouvant se traduire par la perte de quelques éléments binaires dans le récepteur est une microcoupure. Le plus bas niveau auquel puisse être programmé le processeur est la microprogrammation (En microprogramming, De Mikroprogrammierung). On a la microcarte (En microcard, De Mikrofilmkarte), le microcode (En microcode, De Mikro(befehls)code), la microfiche (En microfiche, $D e$ Mikrofiche), le micromodule, la microminiaturisation (En micro-miniaturization, De Mikrominiaturisierung); etc. Le russe utilise le préfixe sous sa forme mikro: mikrokomanda, mikrokod, mikromodul', mikroprogramma, etc. D'autres termes sont moins universels : $E n$ microcolumn $=D e$ Mikrospalte $; n$ micropattern $=D e$ Mikrostruktur; $E n$ microswitch = De Mikroschalter; $F r$ microchaîne, microcomposant, microcopieuse, etc. Cet élément micro joue en opposition avec macroqui signifie grand, gros : micro-instruction $\neq$ macro-instruction, microprocesseur $\neq$ macroprocesseur; microprogrammation $\neq$ macroprogrammation; comme minifonctionne en contraste avec maxi-.

Plus petit que micro-, puisque l'élément veut dire en grec nain, très inférieur à la taille normale, nan(o)-. Placé devant le nom d'une unité, il forme le nom de l'unité un milliard de fois plus petite $\left(10^{-9}\right)$. La nanoseconde (En nanosecond, $D e$ Nanosekunde) est utilisée en informatique (symbole $n$ ); la nanoélectronique est la technologie de la fabrication des circuits intégrés dans lesquels la dimension des éléments se mesure en nanomètres. En médecine, les termes composés de nan $(o)$ - sont nombreux : nanocéphale, nanomèle, etc.

Plus petit encore, on aurait le pico-ordinateur. Dans la connectique nouvelle, «les modules terminaux ne sont pas raccordés individuellement aux différentes unités fonctionnelles; l'unité de gestion devient le centre d'un véritable réseau terminal: elle effectue, sous le contrôle de son mini, micro ou pico-ordinateur selon l'importance de l'installation, l'interconnexion des divers constituants en fonction des services demandés, y compris l'intercommunication ${ }^{3}$. L'élément pic(o)-, tiré de l'italien piccolo, lorsqu'il est placé devant

3. A. Glowinski, Objectif 2000 , p. 159. 
le nom d'une unité, désigne l'unité un million de millions de fois (un billion) plus petite $\left(10^{-12}\right)$; ainsi parle-t-on de picoseconde, picofarad, etc.

Pour désigner la petitesse en espace, temps, volume, quantité, nombre valeur, qualité, etc., le fonds gréco-latin offre au créateur de termes des éléments encore inemployés: parv(i)-, exigu(i)-(exigu, exiguïté), brev(i)- (bréviligne), arct(i)- (arctiligne, coarctation = resserrement), angust(i)- (angustifolié, angustipenné, angustirostre $=$ au bec étroit), infim(i)- (infime, infimité), pauc(i)(pauciflore $=$ qui ne porte que peu de fleurs), pusill(i)- (pusillanime), tenui(ténuirostres = oiseaux à bec très mince), paul(i)-, minut(i)- (menu, minute), brakhy- (brachypnée $=$ respiration courte, brachycéphale), olig(o)- (oligo-éléments, oligarchie, oligurie), élasson(o)-, élakhist(o)-.

Micro- et mini- se situent sur l'échelle des petitesses au même titre que macro- et maxi- sur celle des grandeurs. Ajoutons qu'il existe, fort peu employés encore, pour les deux préfixes provenant du latin: minor(i)- plus petit, moindre (voir minorer $=$ rendre plus petit, moindre; En minor key, De Minoritätladungsträger) opposé à major(i)-, plus grand (voir majorer = rendre plus grand, supérieur; En majority carrier; De Majoritätsladungsträger) et minim( $(i)$-, le plus petit (voir minimum, minimiser; $E n$ minimization, $D e$ Minimierung) opposé à maxim(i)-, le plus grand (voir maximum, maximiser, etc.). Minuscul(i)- $\neq$ majuscul(i)- signifient respectivement : un peu plus petit, assez petit (minuscule) et un peu plus grand, assez grand (majuscule). Les préfixes grecs micr $(o$ )- et macr $(o)$ - ont également donné dans le vocabulaire scientifique deux comparatifs : $m(e ́) i(o)$ - (dans miocène, moins récent) et pli(o)-(dans pliocène, plus récent).

Le degré insuffisant par rapport à la normale et correspondant à la locution adverbiale trop peu, est exprimé par hypo-; la médecine exploite ce préfixe en contraste avec hyper-: hypotrophie, hypertrophie; hypoglycémie, hyperglycémie; hypotension, hypertension. Aussi par sous- $\neq$ sur- et $s u b$ - $\neq$ super-: sous-équipé, sur-équipé, superéquipé. En informatique : un sous-ensemble ( $E n$ subset, De Untermenge); sous-programme (En subroutine, De Subroutine), etc.

Les éléments qui permettent d'exprimer la miniaturisation de plus en plus fine ne manquent donc pas aux informaticiens. Un système terminologique s'instaure; puisse-t-il rester cohérent et clair! 